\title{
Molecular Dynamics and Emerging Network Graphs of In- teractions in Dinitrile-based Li-ion Battery Electrolytes
}

\author{
Thejus. R. Kartha and Bhabani S. Mallik*
}

Department of Chemistry, Indian Institute of Technology Hyderabad, Sangareddy-502285, Telangana, India
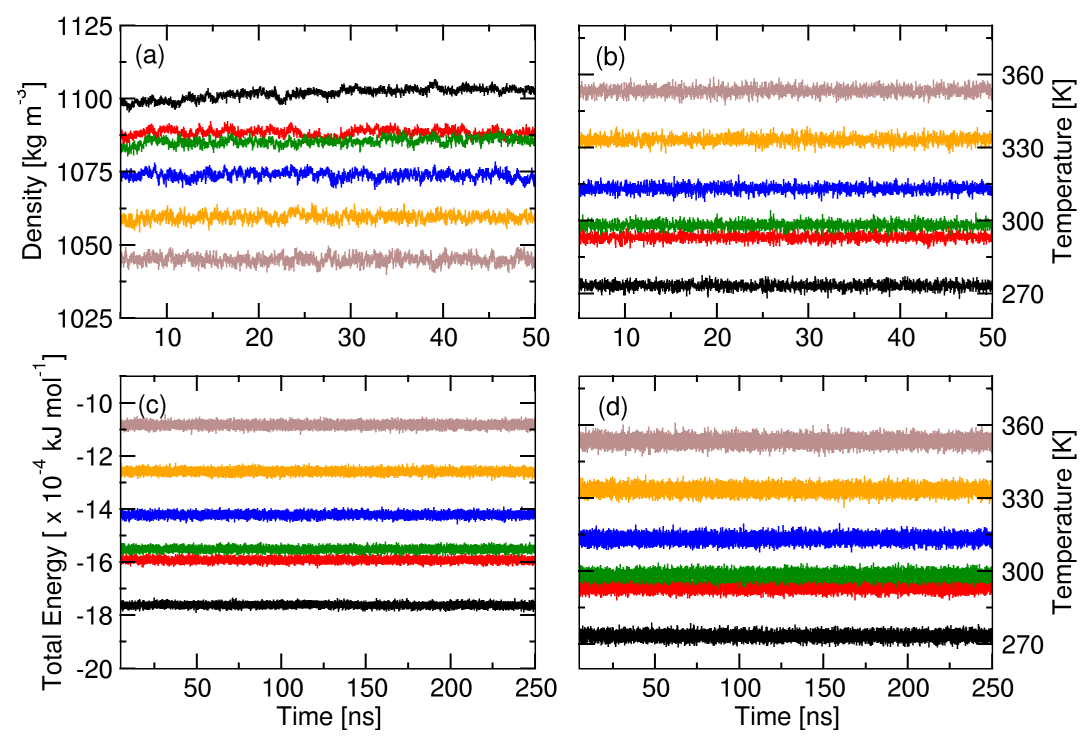

Figure S1. The convergence of physical properties of 1.0 M LiTFSI in ADN simulated at different temperatures. (a) Density with respect to time after NpT, (b) Temperature with respect to time after NVT, (c) Total energy and (d) Temperature with respect to time after NVE simulation.
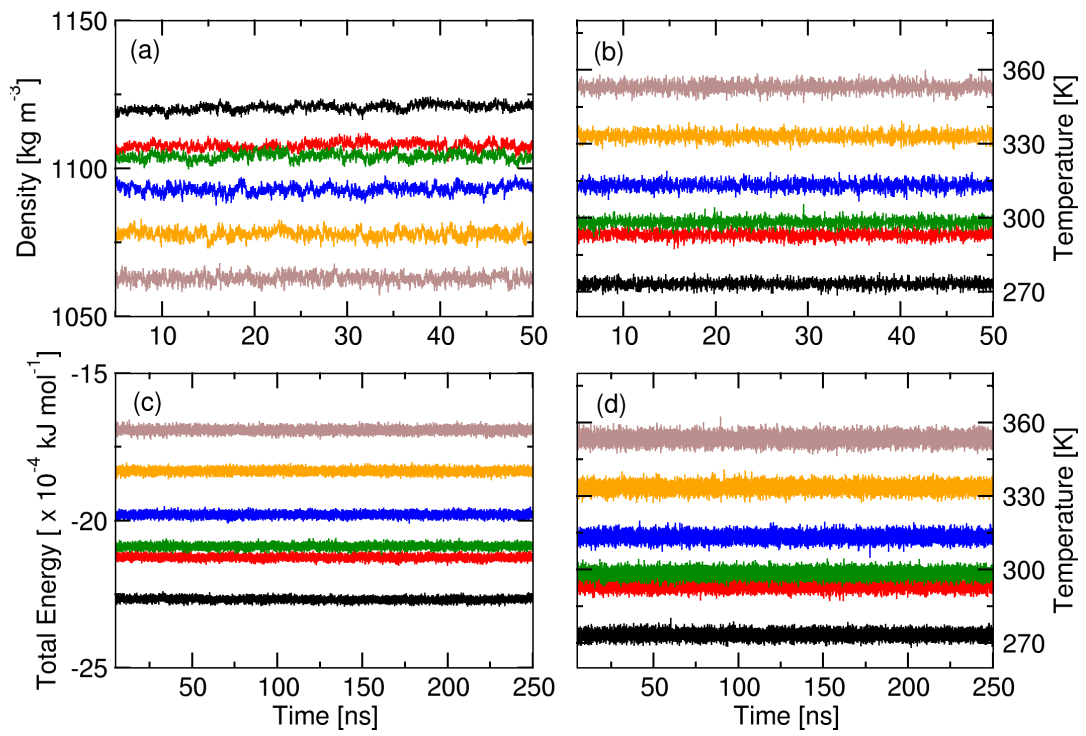

Figure S2. The convergence of physical properties of 1.o M TFSI in GLN simulated at different temperatures. (a) Density with respect to time after NpT, (b) Temperature with respect to time after NVT, (c) Total energy and (d) Temperature with respect to time after NVE simulation. 

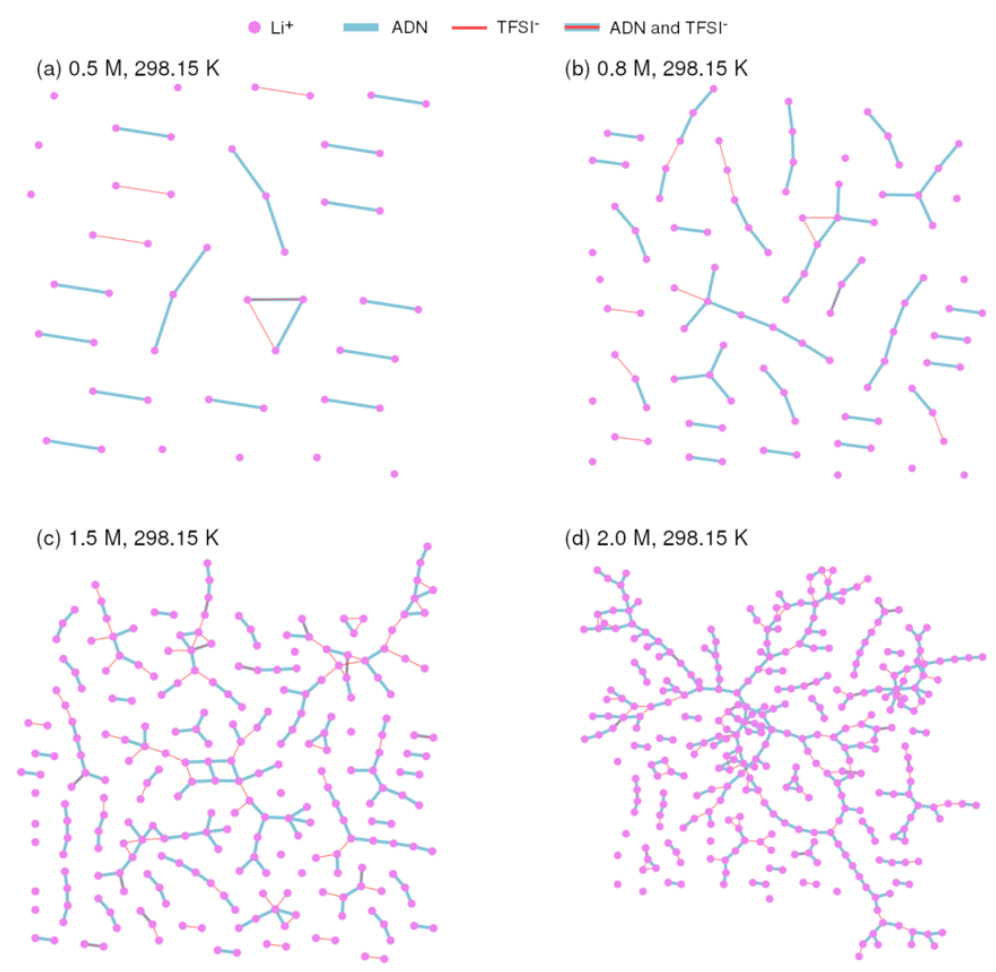

Figure S3. Network graphs of different concentrations of LiTFSI in ADN at 298.15 K.
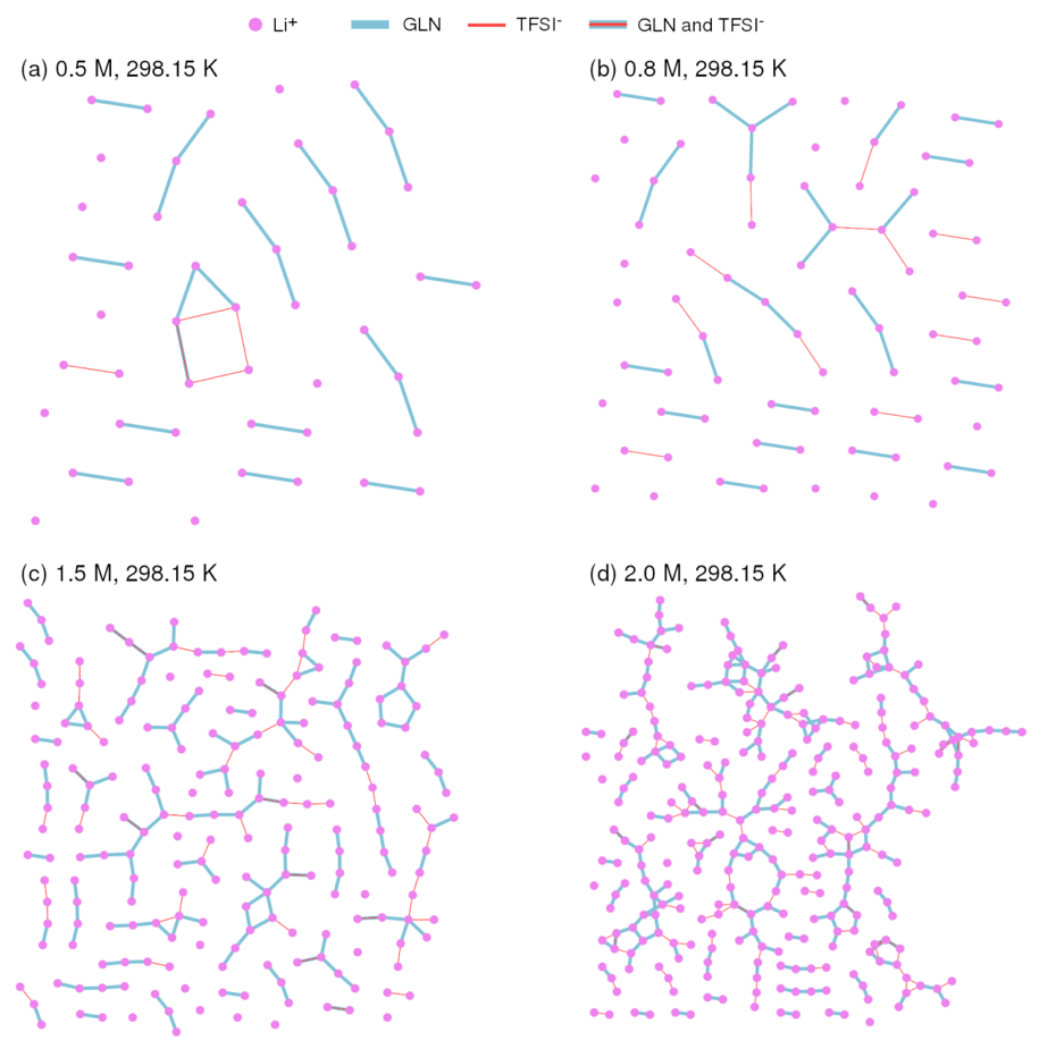

Figure S4. Network graphs of different concentrations of LiTFSI in GLN at 298.15 K. 
- $\mathrm{Li}^{+}=\mathrm{ADN} \longrightarrow \mathrm{TFSI}^{-}=\mathrm{ADN}$ and TFSI
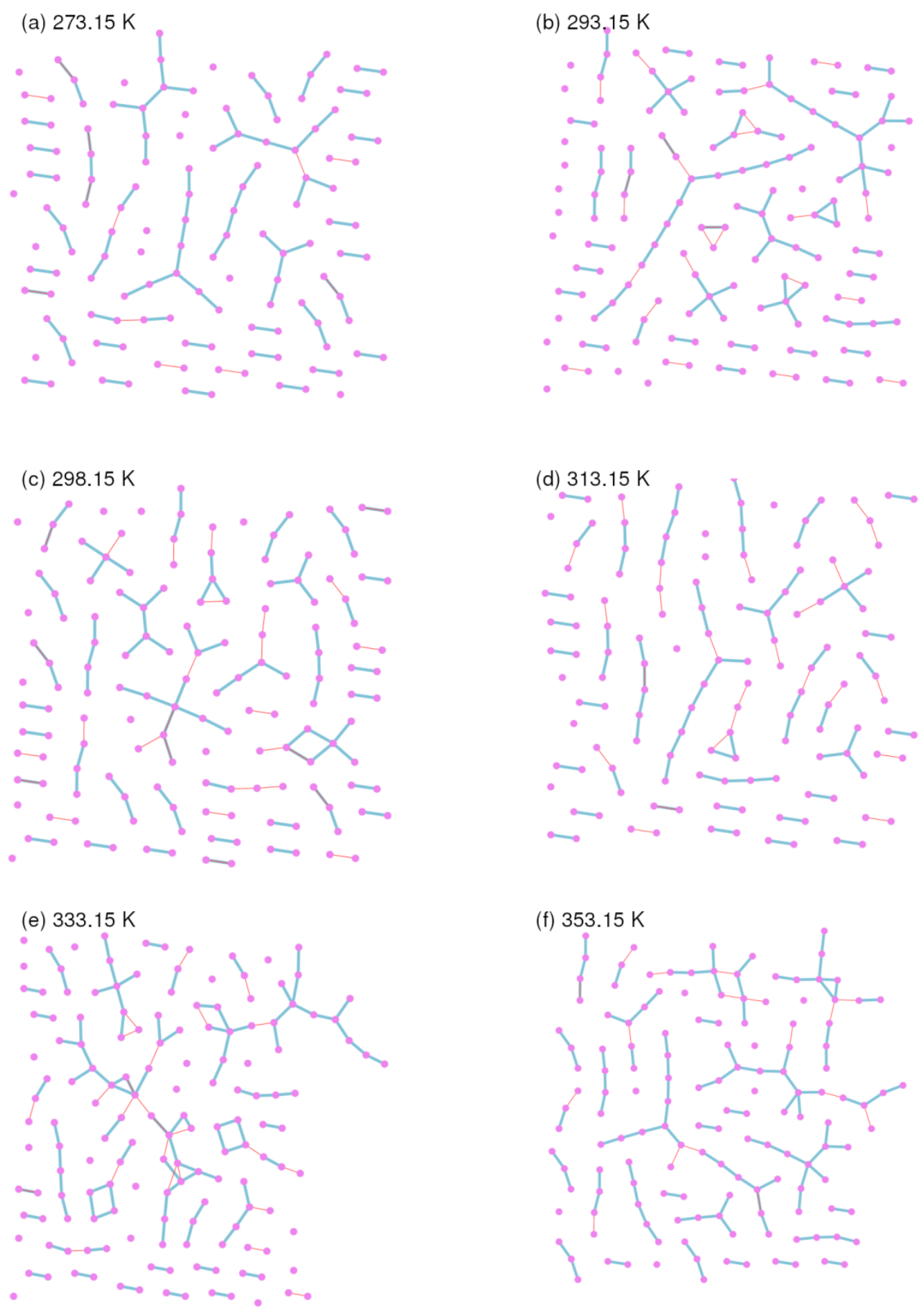

Figure S5. Network graphs of 1 M LiTFSI in ADN at different temperatures. 


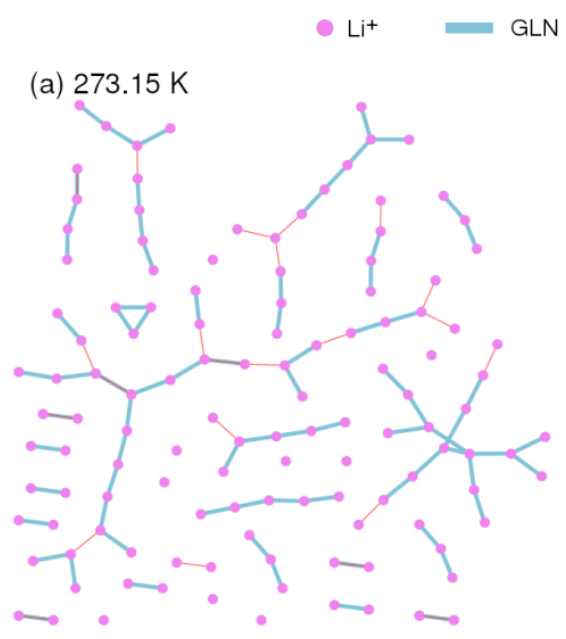

(b) $293.15 \mathrm{~K}$

(c) $298.15 \mathrm{~K}$

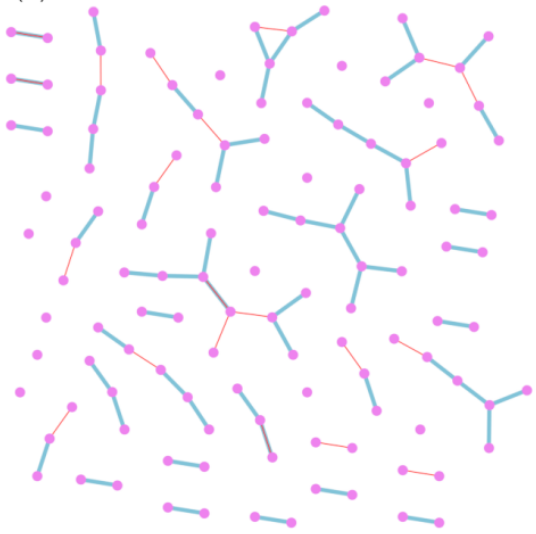

(d) $313.15 \mathrm{~K}$
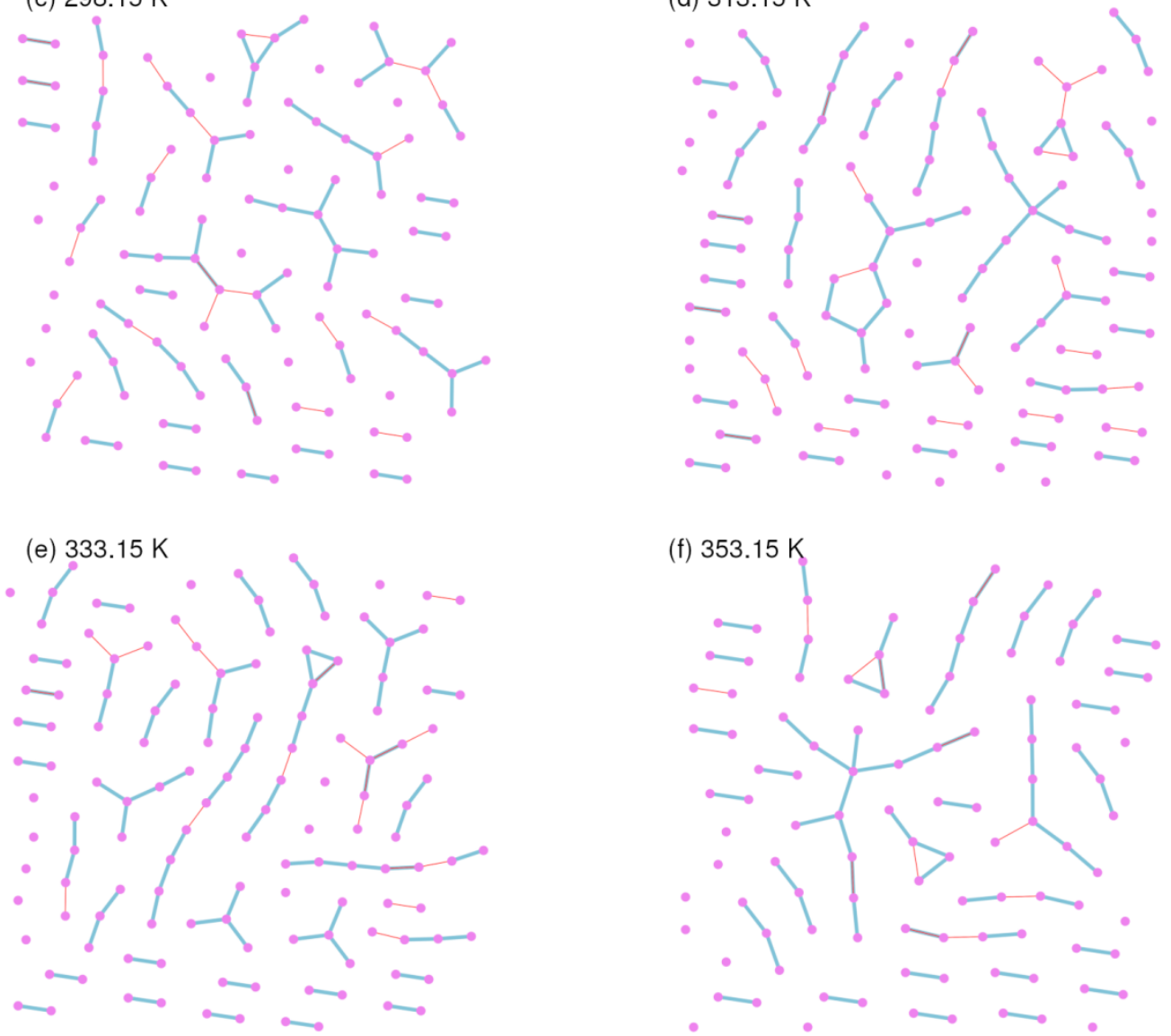

Figure S6. Network graphs of 1 M LiTFSI in GLN at different temperatures. 

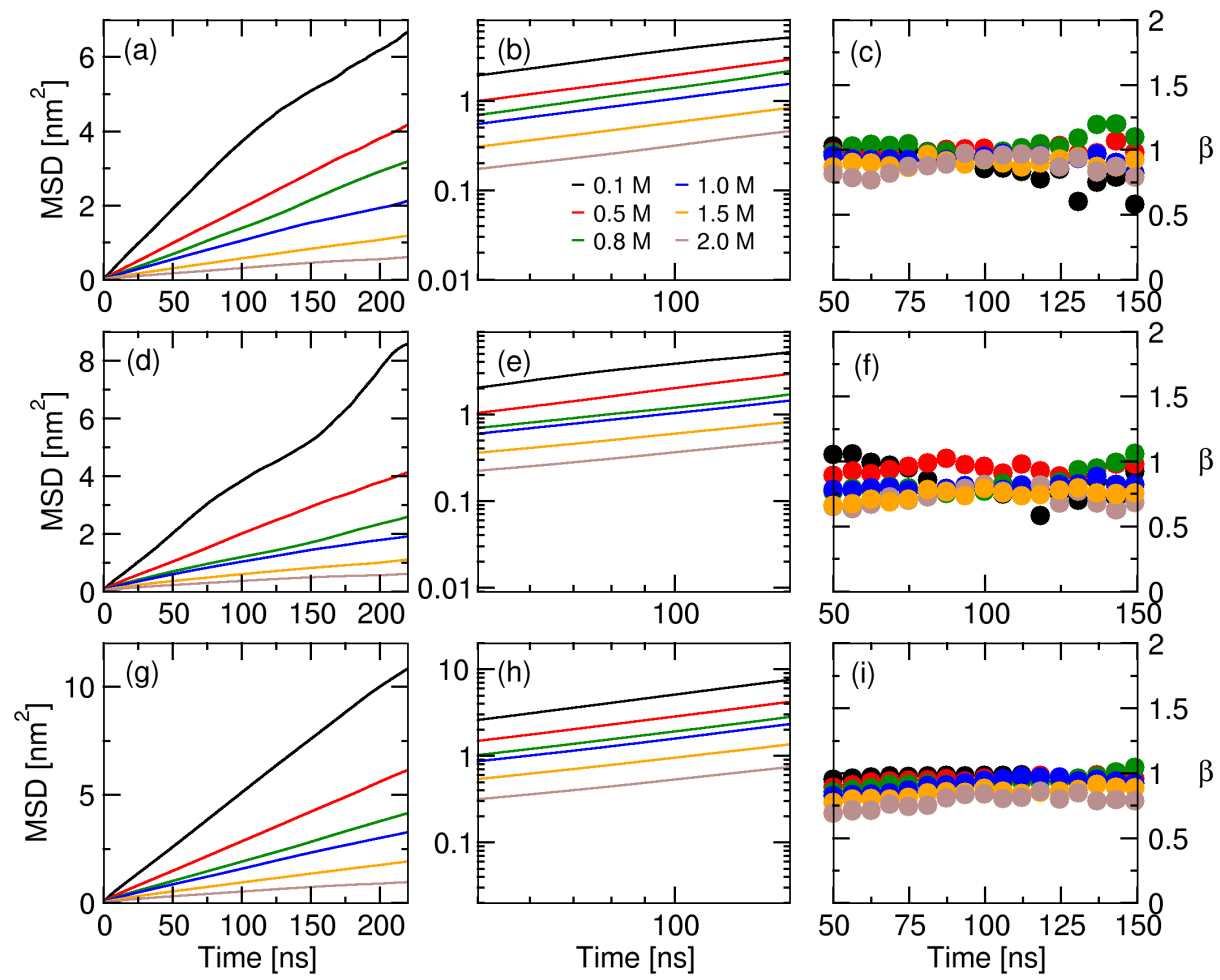

Figure S7. Mean squared displacement, log-log plot of MSD vs. time and $\beta$ vs. time respectively for (a-c) $\mathrm{Li}^{+}$, (df) TFSI- and (g-i) ADN at different concentrations of the mixture.
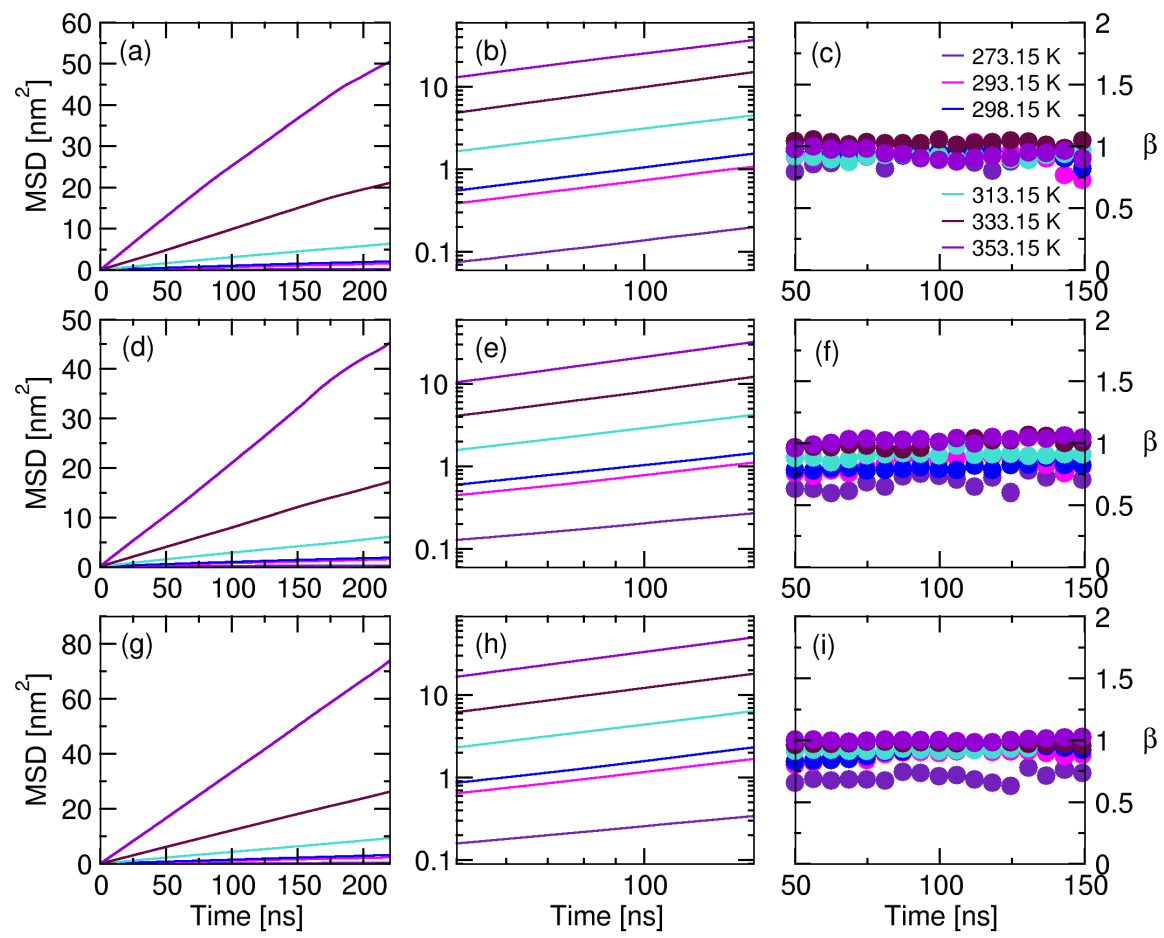

Figure S8. Mean squared displacement, log-log plot of MSD vs. time and $\beta$ vs. time respectively for (a-c) $\mathrm{Li}^{+},(\mathrm{d}-$ f) TFSI- and (g-i) ADN at different temperatures. 

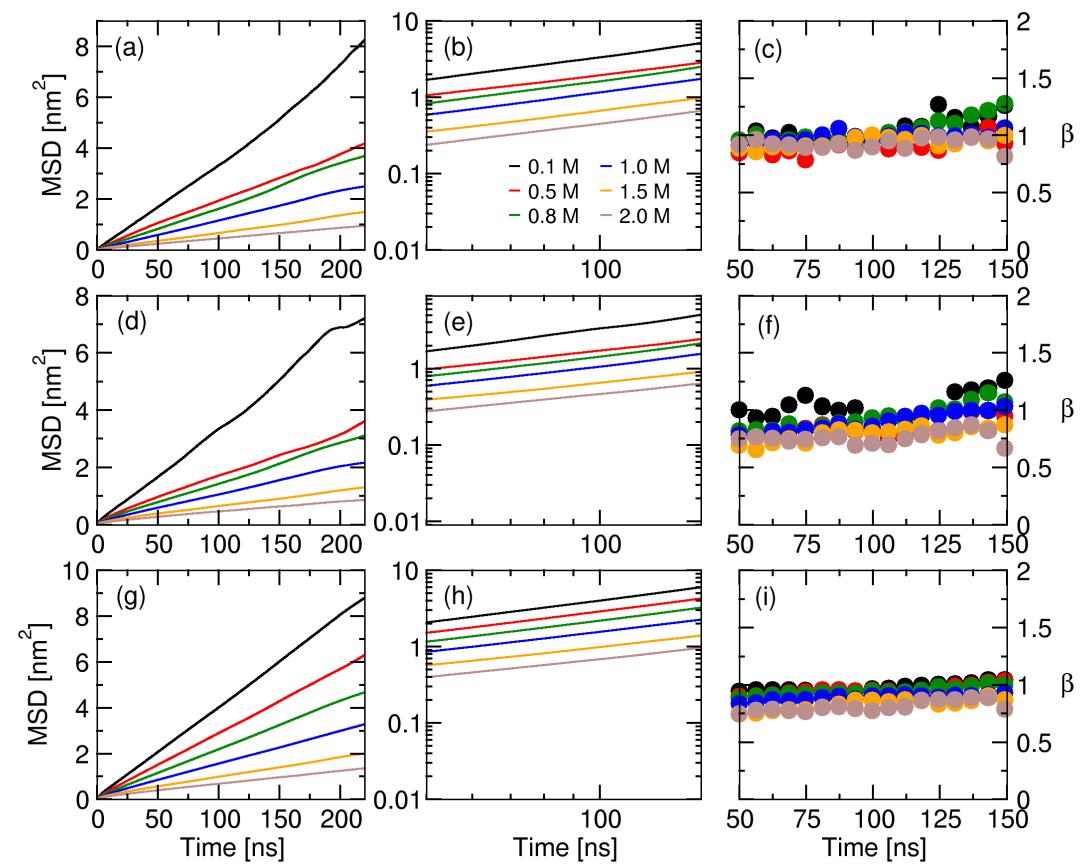

Figure S9. Mean squared displacement, log-log plot of MSD vs. time and $\beta$ vs. time respectively for (a-c) $\mathrm{Li}^{+}$, (d-f) TFSI- and (g-i) GLN at different concentrations of the mixture.
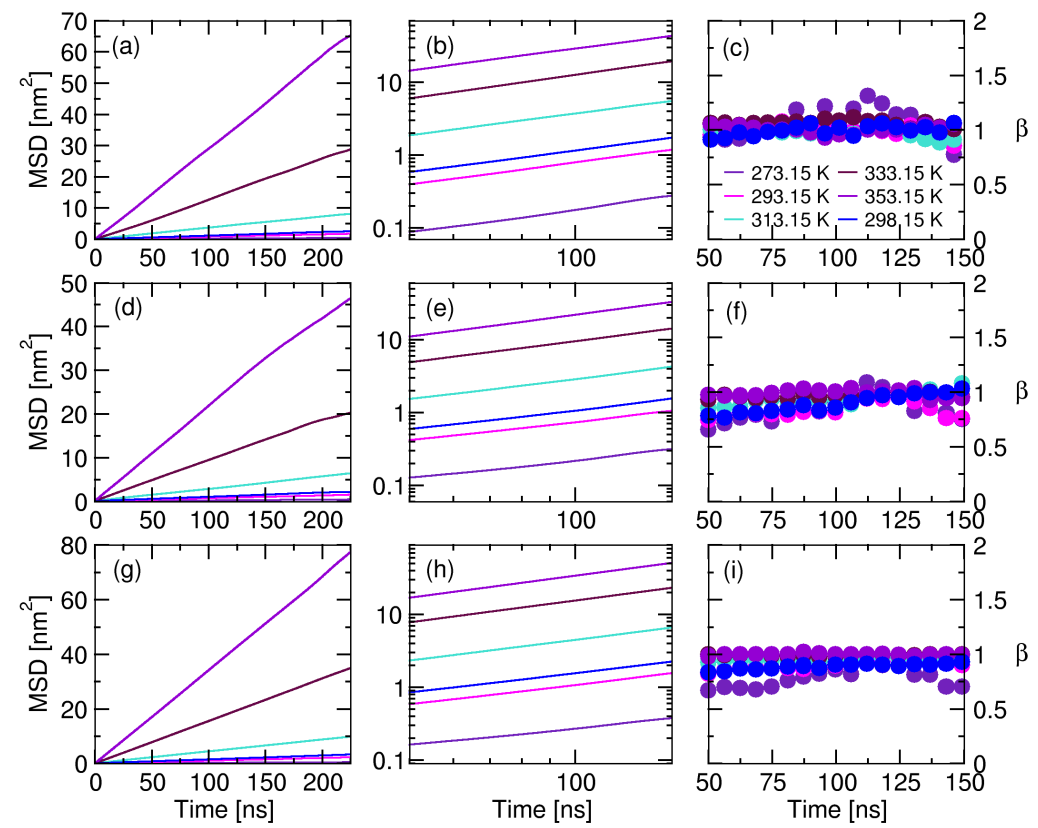

Figure S1o. Mean squared displacement, $\log -\log$ plot of MSD vs. time and $\beta$ vs. time respectively for (a-c) $\mathrm{Li}^{+}$, (d-f) TFSI- and (g-i) GLN at different temperatures. 

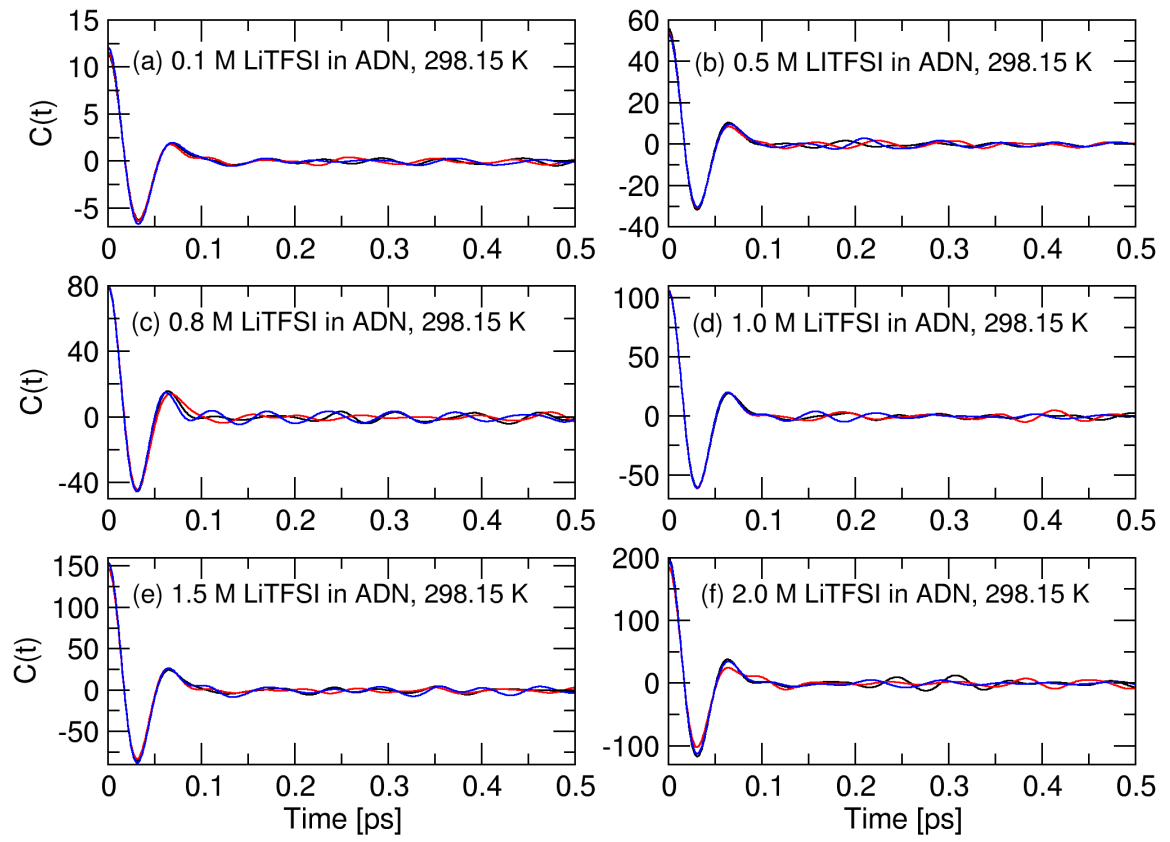

Figure S11. Current autocorrelation functions of different concentrations of LiTFSI in ADN at 298.15 K.
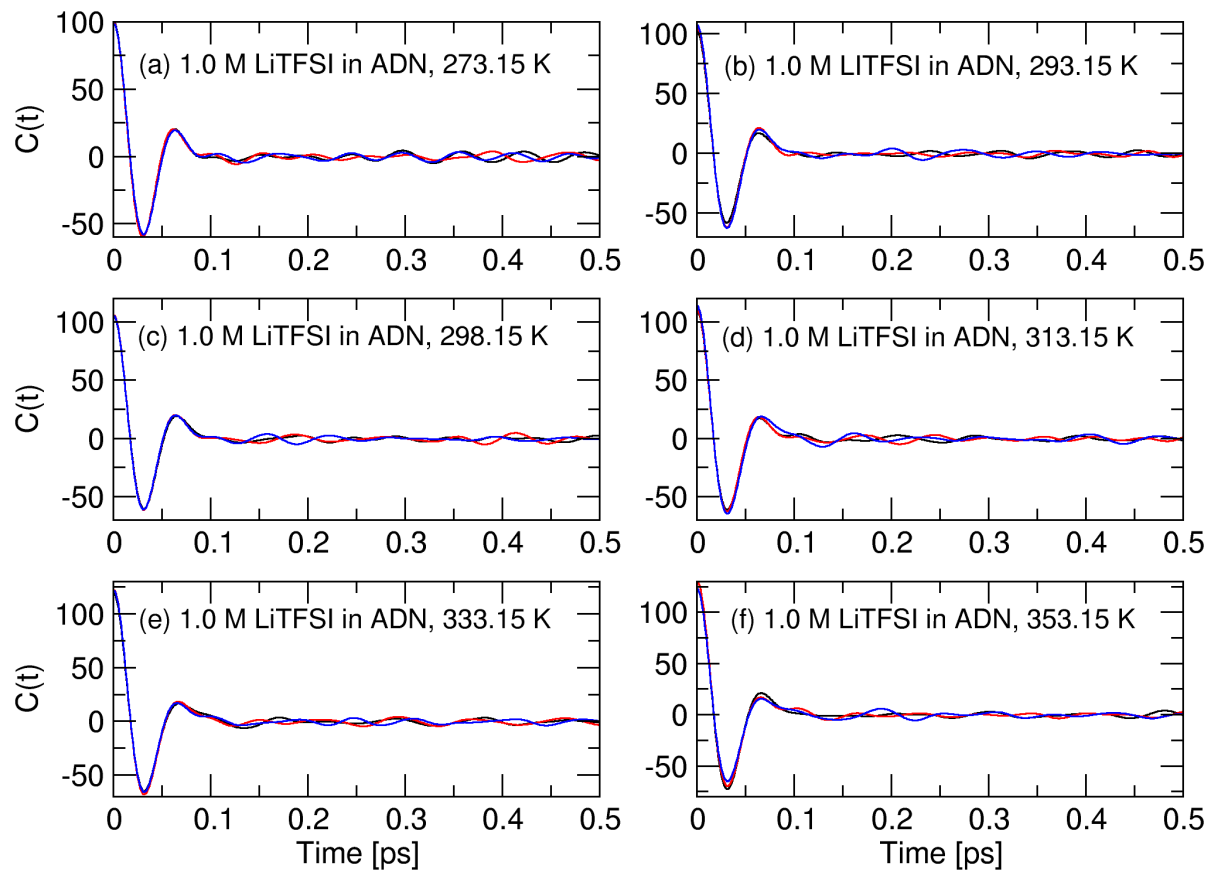

Figure S12. Current autocorrelation functions of 1.0 M LiTFSI in ADN at different temperatures. 

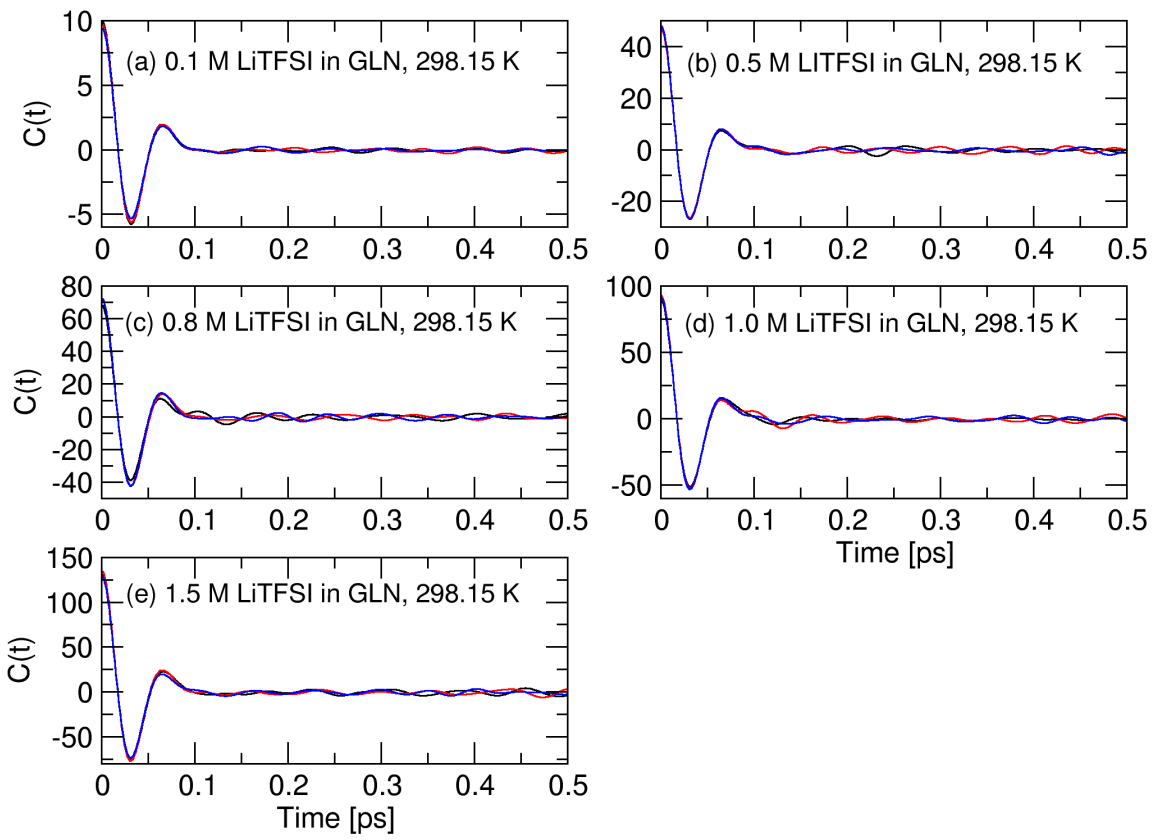

Figure S13. Current autocorrelation functions of different concentrations of LiTFSI in GLN at 298.15 K.
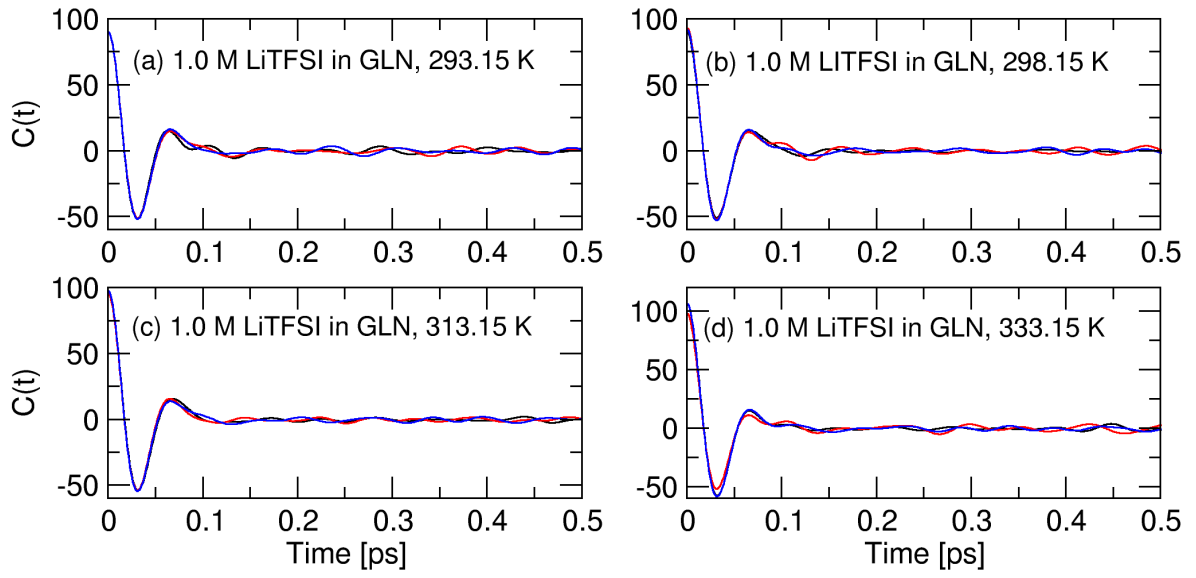

Figure S14. Current autocorrelation functions of 1.0 M LiTFSI in GLN at different temperatures.

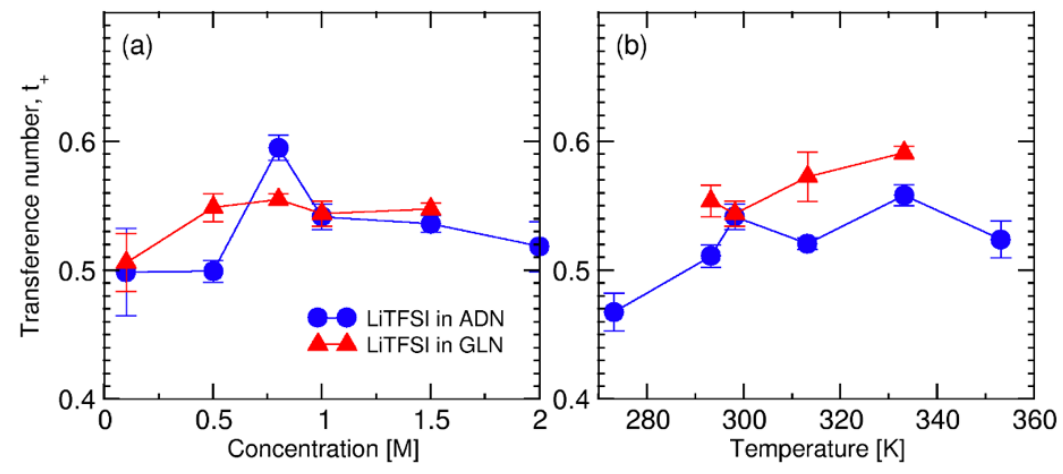

Figure S15. Transference number of the electrolyte with respect to (a) concentration and (b) temperature of of the electrolytes. 
Table S1. Ionic conductivity of different concentrations of LiTFSI in ADN, calculated from current autocorrelation function, cutoff lengths used for integration along with the corresponding experimental values. ${ }^{1,2}$

\begin{tabular}{|c|c|c|c|c|c|c|}
\hline $\begin{array}{l}\text { Concentration of } \\
\text { LiTFSI }[\mathrm{M}]\end{array}$ & $\begin{array}{c}\text { Temperature } \\
{[\mathrm{K}]}\end{array}$ & $\begin{array}{l}\text { Cutoff } \\
\text { [ps] }\end{array}$ & $\begin{array}{l}\text { Ionic conductivity } \\
{\left[\mathrm{mS} \mathrm{cm}^{-1}\right]}\end{array}$ & Average & $\begin{array}{l}\text { Standard } \\
\text { deviation }\end{array}$ & $\begin{array}{c}\text { Experimental ionic } \\
\text { conductivity }\left[\mathrm{mS} \mathrm{cm}^{-1}\right]\end{array}$ \\
\hline \multirow{3}{*}{0.1} & \multirow{3}{*}{298.15} & 10.915 & 0.2963 & \multirow{3}{*}{0.3435} & \multirow{3}{*}{0.09192} & \multirow{3}{*}{0.487} \\
\hline & & 11.17 & 0.2622 & & & \\
\hline & & 11.12 & 0.472 & & & \\
\hline \multirow{3}{*}{0.5} & \multirow{3}{*}{298.15} & 11.11 & 1.8898 & \multirow{3}{*}{2.0021} & \multirow{3}{*}{0.0853} & \multirow{3}{*}{2.022} \\
\hline & & 10.905 & 2.0203 & & & \\
\hline & & 11.17 & 2.0963 & & & \\
\hline \multirow{3}{*}{0.8} & \multirow{3}{*}{298.15} & 10.935 & 1.9638 & \multirow{3}{*}{2.2423} & \multirow{3}{*}{0.2057} & \multirow{3}{*}{2.335} \\
\hline & & 10.9 & 2.3087 & & & \\
\hline & & 10.96 & 2.4545 & & & \\
\hline \multirow{3}{*}{1.0} & \multirow{3}{*}{298.15} & 11.195 & 2.2733 & \multirow{3}{*}{2.3391} & \multirow{3}{*}{0.0520} & \multirow{3}{*}{2.382} \\
\hline & & 11.14 & 2.4006 & & & \\
\hline & & 11.12 & 2.3434 & & & \\
\hline \multirow{3}{*}{1.5} & \multirow{3}{*}{298.15} & 11.155 & 1.7396 & \multirow{3}{*}{1.763} & \multirow{3}{*}{0.0183} & \multirow{3}{*}{1.775} \\
\hline & & 11.18 & 1.7651 & & & \\
\hline & & 11.19 & 1.7843 & & & \\
\hline \multirow{3}{*}{2.0} & \multirow{3}{*}{298.15} & 11.08 & 1.1579 & \multirow{3}{*}{1.1938} & \multirow{3}{*}{0.0457} & \multirow{3}{*}{1.207} \\
\hline & & 11.04 & 1.1653 & & & \\
\hline & & 11.095 & 1.2583 & & & \\
\hline
\end{tabular}

Table S2. Ionic conductivity of different concentrations of LiTFSI in GLN, calculated from current autocorrelation function, cutoff lengths used for integration along with the corresponding experimental values. ${ }^{1,2}$

\begin{tabular}{|c|c|c|c|c|c|c|}
\hline $\begin{array}{l}\text { Concentration of } \\
\text { LiTFSI }[\mathrm{M}]\end{array}$ & $\begin{array}{c}\text { Temperature } \\
{[\mathrm{K}]}\end{array}$ & $\begin{array}{l}\text { Cutoff } \\
\text { [ps] }\end{array}$ & $\begin{array}{l}\text { Ionic conductivity } \\
\qquad\left[\mathrm{mS} \mathrm{cm}^{-1}\right]\end{array}$ & Average & $\begin{array}{l}\text { Standard } \\
\text { deviation }\end{array}$ & $\begin{array}{c}\text { Experimental ionic } \\
\text { conductivity }\left[\mathrm{mS} \mathrm{cm}^{-1}\right]\end{array}$ \\
\hline \multirow{3}{*}{0.1} & \multirow{3}{*}{298.15} & 11.42 & 0.1835 & \multirow{3}{*}{0.1844} & \multirow{3}{*}{0.0022} & \multirow{3}{*}{0.182} \\
\hline & & 11.445 & 0.1822 & & & \\
\hline & & 11.31 & 0.1874 & & & \\
\hline \multirow{3}{*}{0.5} & \multirow{3}{*}{298.15} & 11.61 & 1.9634 & \multirow{3}{*}{1.8596} & \multirow{3}{*}{0.0734} & \multirow{3}{*}{1.962} \\
\hline & & 11.565 & 1.8119 & & & \\
\hline & & 11.63 & 1.8036 & & & \\
\hline \multirow{3}{*}{0.8} & \multirow{3}{*}{298.15} & 11.4 & 2.5128 & \multirow{3}{*}{2.3605} & \multirow{3}{*}{0.1821} & \multirow{3}{*}{2.437} \\
\hline & & 11.335 & 2.4643 & & & \\
\hline & & 11.54 & 2.1045 & & & \\
\hline \multirow{3}{*}{1.0} & \multirow{3}{*}{298.15} & 11.405 & 2.4768 & \multirow{3}{*}{2.5147} & \multirow{3}{*}{0.0363} & \multirow{3}{*}{2.500} \\
\hline & & 11.225 & 2.5636 & & & \\
\hline & & 11.695 & 2.5037 & & & \\
\hline \multirow{3}{*}{1.5} & \multirow{3}{*}{298.15} & 11.445 & 1.7912 & \multirow{3}{*}{1.7771} & \multirow{3}{*}{0.0174} & \multirow{3}{*}{1.775} \\
\hline & & 11.405 & 1.7526 & & & \\
\hline & & 11.475 & 1.7875 & & & \\
\hline
\end{tabular}


Table S3. Ionic conductivity of LiTFSI in ADN at different temperatures, calculated from current autocorrelation function, cutoff lengths used for integration along with the corresponding experimental values. ${ }^{1,2}$

\begin{tabular}{|c|c|c|c|c|c|c|}
\hline $\begin{array}{l}\text { Concentration } \\
\text { of LiTFSI [M] }\end{array}$ & $\begin{array}{c}\text { Temperature } \\
{[\mathrm{K}]}\end{array}$ & $\begin{array}{l}\text { Cutoff } \\
\text { [ps] }\end{array}$ & $\begin{array}{c}\text { Ionic conductiv- } \\
\text { ity } \\
{\left[\mathrm{mS} \mathrm{cm}^{-1}\right]} \\
\end{array}$ & Average & $\begin{array}{l}\text { Standard } \\
\text { deviation }\end{array}$ & $\begin{array}{c}\text { Experimental ionic } \\
\text { conductivity }[\mathrm{mS} \\
\left.\mathrm{cm}^{-1}\right]\end{array}$ \\
\hline \multirow{3}{*}{1.0} & \multirow{3}{*}{273.15} & 11.17 & 0.7526 & \multirow{3}{*}{0.7725} & \multirow{3}{*}{0.0151} & \multirow{3}{*}{0.770} \\
\hline & & 11.185 & 0.7891 & & & \\
\hline & & 11.16 & 0.7759 & & & \\
\hline \multirow{3}{*}{1.0} & \multirow{3}{*}{293.15} & 11.105 & 2.1281 & \multirow{3}{*}{2.1213} & \multirow{3}{*}{0.0077} & \multirow{3}{*}{2.010} \\
\hline & & 11.02 & 2.1105 & & & \\
\hline & & 11.125 & 2.1253 & & & \\
\hline \multirow{3}{*}{1.0} & \multirow{3}{*}{298.15} & 11.195 & 2.2733 & \multirow{3}{*}{2.3391} & \multirow{3}{*}{0.0520} & \multirow{3}{*}{2.382} \\
\hline & & 11.14 & 2.4006 & & & \\
\hline & & 11.12 & 2.3434 & & & \\
\hline \multirow{3}{*}{1.0} & \multirow{3}{*}{313.15} & 10.995 & 3.4227 & \multirow{3}{*}{3.4336} & \multirow{3}{*}{0.2675} & \multirow{3}{*}{3.660} \\
\hline & & 11.08 & 3.7665 & & & \\
\hline & & 10.905 & 3.1115 & & & \\
\hline \multirow{3}{*}{1.0} & \multirow{3}{*}{333.15} & 10.955 & 5.7621 & \multirow{3}{*}{4.5327} & \multirow{3}{*}{0.8718} & \multirow{3}{*}{$5 \cdot 740$} \\
\hline & & 10.94 & 3.8361 & & & \\
\hline & & 11.145 & 4.0002 & & & \\
\hline \multirow{3}{*}{1.0} & \multirow{3}{*}{353.15} & 11.02 & 4.1524 & \multirow{3}{*}{5.0710} & \multirow{3}{*}{0.7544} & \multirow{3}{*}{8.090} \\
\hline & & 8.6 & 6.0001 & & & \\
\hline & & 11.7 & 5.0605 & & & \\
\hline
\end{tabular}

Table S4. Ionic conductivity of LiTFSI in GLN at different temperatures, calculated from current autocorrelation function, cutoff lengths used for integration along with the corresponding experimental values. ${ }^{1,2}$

\begin{tabular}{|c|c|c|c|c|c|c|}
\hline $\begin{array}{l}\text { Concentration } \\
\text { of LiTFSI [M] }\end{array}$ & $\begin{array}{c}\text { Temperature } \\
{[\mathrm{K}]}\end{array}$ & $\begin{array}{l}\text { Cutoff } \\
\text { [ps] }\end{array}$ & $\begin{array}{c}\text { Ionic conductiv- } \\
\text { ity } \\
{\left[\mathrm{mS} \mathrm{cm}^{-1}\right]}\end{array}$ & Average & $\begin{array}{l}\text { Standard } \\
\text { deviation }\end{array}$ & $\begin{array}{c}\text { Experimental ionic } \\
\text { conductivity }[\mathrm{mS} \\
\left.\mathrm{cm}^{-1}\right]\end{array}$ \\
\hline \multirow{3}{*}{1.0} & \multirow{3}{*}{293.15} & 11.355 & 1.967 & \multirow{3}{*}{1.9794} & \multirow{3}{*}{0.0105} & \multirow{3}{*}{1.998} \\
\hline & & 11.435 & 1.9785 & & & \\
\hline & & 11.415 & 1.9926 & & & \\
\hline \multirow{3}{*}{1.0} & \multirow{3}{*}{298.15} & 11.405 & 2.4768 & \multirow{3}{*}{2.5147} & \multirow{3}{*}{0.0363} & \multirow{3}{*}{2.500} \\
\hline & & 11.225 & 2.5636 & & & \\
\hline & & 11.695 & 2.5037 & & & \\
\hline \multirow{3}{*}{1.0} & \multirow{3}{*}{313.15} & 11.225 & 1.9469 & \multirow{3}{*}{3.0179} & \multirow{3}{*}{0.7573} & \multirow{3}{*}{$3 \cdot 512$} \\
\hline & & 11.615 & 3.546 & & & \\
\hline & & 11.66 & 3.5607 & & & \\
\hline \multirow{3}{*}{1.0} & \multirow{3}{*}{333.15} & 11.69 & 5.1756 & \multirow{3}{*}{4.2996} & \multirow{3}{*}{0.9374} & \multirow{3}{*}{$5 \cdot 506$} \\
\hline & & 11.2 & 4.7233 & & & \\
\hline & & 11.645 & 2.9998 & & & \\
\hline
\end{tabular}

\section{REFERENCES}

(1) Farhat, D.; Ghamouss, F.; Maibach, J.; Edström, K.; Lemordant, D. Adiponitrile-Lithium Bis(Trimethylsulfonyl)Imide Solutions as Alkyl Carbonate-Free Electrolytes for Li4Ti5O12 (LTO)/LiNi1/3Co1/3Mn1/3O2 (NMC) Li-Ion Batteries. ChemPhysChem 2o17, 18 (10), 1333-1344. https://doi.org/10.1002/cphc.201700058.

(2) Farhat, D.; Lemordant, D.; Jacquemin, J.; Ghamouss, F. Alternative Electrolytes for Li-Ion Batteries Using Glutaronitrile and 2-Methylglutaronitrile with Lithium Bis(Trifluoromethanesulfonyl) Imide. J. Electrochem. Soc. 2019, 166 (14), A3487. https://doi.org/10.1149/2.1261914jes. 


\section{Non-Bonded Force Field Parameters}
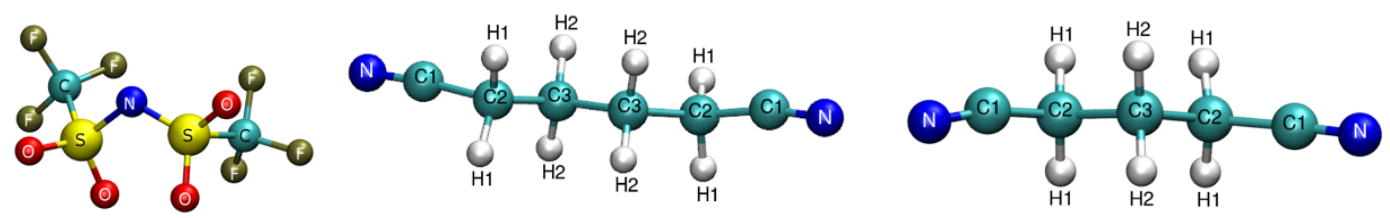

\begin{tabular}{|c|c|c|c|}
\hline Atomtype & $\sigma[\mathbf{n m}]$ & $\varepsilon\left[\mathrm{kJ} \mathrm{mol}^{-1}\right]$ & Charge (unscaled) \\
\hline $\mathbf{L i}$ & 0.2337 & 0.67000 & +1.000000 \\
\hline $\mathbf{N}_{\text {TFSI }}$ & 0.325000 & 0.711280 & -0.846520 \\
\hline $\mathbf{S}_{\text {TFSI }}$ & 0.356359 & 1.046000 & +1.327211 \\
\hline $\mathbf{O}_{\text {TFSI }}$ & 0.295992 & 0.878640 & -0.623515 \\
\hline CTFSI & 0.339967 & 0.457730 & +0.204906 \\
\hline F TFSI & 0.311815 & 0.255224 & -0.120609 \\
\hline $\mathbf{N}_{\text {ADN }}$ & 0.325000 & 0.711280 & -0.484949 \\
\hline C1ADN & 0.339967 & 0.878640 & +0.356624 \\
\hline C2ADN & 0.339967 & 0.457730 & -0.140183 \\
\hline C3ADN & 0.339967 & 0.457730 & +0.035247 \\
\hline H1 AdN & 0.264953 & 0.0656888 & +0.090271 \\
\hline H2 & 0.264953 & 0.0656888 & +o.026359 \\
\hline NGLN & 0.325000 & 0.711280 & -0.471701 \\
\hline C1GLn & 0.339967 & 0.878640 & +0.342369 \\
\hline C2GLN & 0.339967 & 0.457730 & -0.140507 \\
\hline C3GLN & 0.339967 & 0.457730 & +0.081522 \\
\hline H1GLN & 0.264953 & 0.0656888 & +0.094445 \\
\hline H2GLN & 0.264953 & 0.0656888 & +0.040188 \\
\hline
\end{tabular}




\section{Bonded Force Field Parameters}

(Topology files for GROMACS, excluding charge scaling factors)

\section{TFSI}

\begin{tabular}{|c|c|c|c|c|c|c|c|c|}
\hline \multicolumn{9}{|c|}{ [ atoms ] } \\
\hline \multirow[t]{16}{*}{; } & $\mathrm{nr}$ & type & resi & res & atom & cgnr & charge & mass \\
\hline & 1 & $f$ & 1 & TFS & F1 & 1 & -0.120609 & 19.00000 \\
\hline & 2 & c3 & 1 & TFS & $\mathrm{C} 1$ & 2 & 0.204906 & 12.01000 \\
\hline & 3 & $\mathrm{f}$ & 1 & TFS & F2 & 3 & -0.120609 & 19.00000 \\
\hline & 4 & $\mathrm{f}$ & 1 & TFS & F3 & 4 & -0.120609 & 19.00000 \\
\hline & 5 & sy & 1 & TFS & S1 & 5 & 1.327211 & 32.06000 \\
\hline & 6 & 0 & 1 & TFS & 03 & 6 & -0.623515 & 16.00000 \\
\hline & 7 & $\circ$ & 1 & TFS & 04 & 7 & -0.623515 & 16.00000 \\
\hline & 8 & ne & 1 & TFS & N1 & 8 & -0.846520 & 14.01000 \\
\hline & 9 & s6 & 1 & TFS & s2 & 9 & 1.327211 & 32.06000 \\
\hline & 10 & 0 & 1 & TFS & 01 & 10 & -0.623515 & 16.00000 \\
\hline & 11 & 0 & 1 & TFS & 02 & 11 & -0.623515 & 16.00000 \\
\hline & 12 & c3 & 1 & TFS & $\mathrm{C} 2$ & 12 & 0.204906 & 12.01000 \\
\hline & 13 & $\mathrm{f}$ & 1 & TFS & F5 & 13 & -0.120609 & 19.00000 \\
\hline & 14 & $f$ & 1 & TFS & $\mathrm{F} 6$ & 14 & -0.120609 & 19.00000 \\
\hline & 15 & $\mathrm{f}$ & 1 & TFS & F4 & 15 & -0.120609 & 19.00000 \\
\hline \multicolumn{9}{|c|}{ [ bonds ] } \\
\hline & ai & $a j$ & funct & \multicolumn{3}{|l|}{ r } & \multicolumn{2}{|l|}{$\mathrm{k}$} \\
\hline & 1 & 2 & 1 & \multicolumn{2}{|c|}{$1.3497 e-01$} & & $2.9865 e+05$ & $\mathrm{~F} 1-\mathrm{C} 1$ \\
\hline & 2 & 3 & 1 & \multicolumn{2}{|c|}{$1.3497 e-01$} & & $2.9865 e+05 ;$ & $\mathrm{C} 1-\mathrm{F} 2$ \\
\hline & 2 & 4 & 1 & \multicolumn{2}{|c|}{$1.3497 e-01$} & & $2.9865 e+05$ & $\mathrm{C} 1-\mathrm{F} 3$ \\
\hline & 2 & 5 & 1 & \multicolumn{2}{|c|}{$1.8087 e-01$} & & $1.9481 e+05$ & $\mathrm{C} 1-\mathrm{S} 1$ \\
\hline & 5 & 6 & 1 & \multicolumn{2}{|c|}{$1.4660 \mathrm{e}-01$} & & & S1 - 03 \\
\hline & 5 & 7 & 1 & \multicolumn{2}{|c|}{$1.4660 \mathrm{e}-01$} & & $\begin{array}{l}4.1254 \mathrm{e}+05 ; \\
4.1254 \mathrm{e}+05 ;\end{array}$ & $\mathrm{S} 1-04$ \\
\hline & 5 & 8 & 1 & \multicolumn{2}{|c|}{$1.6723 e-01$} & & $2.6535 \mathrm{e}+05:$ & $\mathrm{S} 1-\mathrm{N} 1$ \\
\hline & 8 & 9 & 1 & \multicolumn{2}{|c|}{$1.5540 \mathrm{e}-01$} & \multicolumn{2}{|r|}{$3.6928 \mathrm{e}+05$} & $\mathrm{~N} 1-\mathrm{S} 2$ \\
\hline & 9 & 10 & 1 & \multicolumn{2}{|c|}{$1.4533 e-01$} & & $4.2903 e+05$ & $\mathrm{~S} 2-01$ \\
\hline & 9 & 11 & 1 & \multicolumn{2}{|c|}{$1.4533 \mathrm{e}-01$} & & $4.2903 e+05:$ & $\mathrm{s} 2-02$ \\
\hline & 9 & 12 & 1 & \multicolumn{2}{|c|}{$1.8075 e-01$} & & $1.9539 e+05 ;$ & $\mathrm{S} 2-\mathrm{C} 2$ \\
\hline & 12 & 13 & 1 & 1.3 & $497 e-01$ & & $2.9865 e+05 ;$ & $\mathrm{C} 2-\mathrm{F} 5$ \\
\hline & 12 & 14 & 1 & 1.3 & $497 e-01$ & & $2.9865 e+05$ & $\mathrm{C} 2-\mathrm{F} 6$ \\
\hline & 12 & 15 & 1 & 1.3 & $497 e-01$ & & $2.9865 e+05$ & $\mathrm{C} 2-\mathrm{F} 4$ \\
\hline
\end{tabular}

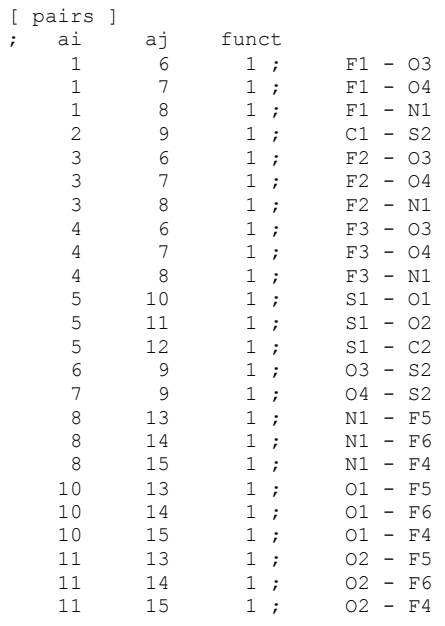

\begin{tabular}{|c|c|c|c|c|c|c|c|c|c|}
\hline \multicolumn{10}{|c|}{ angles ] } \\
\hline$a i$ & $a j$ & $a k$ & funct & theta & cth & & & & \\
\hline 1 & 2 & 3 & 1 & $1.0736 \mathrm{e}+02$ & $5.9321 \mathrm{e}+02$ & ; & F1 & $-\mathrm{C} 1$ & - F2 \\
\hline 1 & 2 & 4 & 1 & $1.0736 \mathrm{e}+02$ & $5.9321 e+02$ & ; & F1 & $-\mathrm{C} 1$ & $-F 3$ \\
\hline 1 & 2 & 5 & 1 & $1.0968 \mathrm{e}+02$ & $5.2819 e+02$ & ; & F1 & $-\mathrm{C} 1$ & $-\mathrm{S} 1$ \\
\hline 2 & 5 & 6 & 1 & $1.0785 e+02$ & $5.4752 e+02$ & ; & $\mathrm{C} 1$ & $-\mathrm{s} 1$ & -03 \\
\hline 2 & 5 & 7 & 1 & $1.0785 e+02$ & $5.4752 e+02$ & ; & $\mathrm{C} 1$ & $-\mathrm{s} 1$ & -04 \\
\hline 2 & 5 & 8 & 1 & $1.0219 \mathrm{e}+02$ & $5.3614 \mathrm{e}+02$ & ; & $\mathrm{C} 1$ & $-\mathrm{s} 1$ & - N1 \\
\hline 3 & 2 & 4 & 1 & $1.0736 \mathrm{e}+02$ & $5.9321 \mathrm{e}+02$ & ; & F2 & $-\mathrm{C} 1$ & $-F 3$ \\
\hline 3 & 2 & 5 & 1 & $1.0968 e+02$ & $5.2819 e+02$ & ; & F2 & $-\mathrm{C} 1$ & $-\mathrm{S} 1$ \\
\hline 4 & 2 & 5 & 1 & $1.0968 e+02$ & $5.2819 e+02$ & ; & F3 & $-\mathrm{C} 1$ & $-\mathrm{S} 1$ \\
\hline 5 & 8 & 9 & 1 & $1.1918 \mathrm{e}+02$ & $5.7220 e+02$ & ; & S1 & $-\mathrm{N} 1$ & $-\mathrm{s} 2$ \\
\hline 6 & 5 & 7 & 1 & $1.2141 \mathrm{e}+02$ & $6.0701 e+02$ & ; & 03 & $-\mathrm{s} 1$ & -04 \\
\hline 6 & 5 & 8 & 1 & $1.0965 e+02$ & $5.8827 e+02$ & ; & 03 & $-\mathrm{s} 1$ & $-\mathrm{N} 1$ \\
\hline 7 & 5 & 8 & 1 & $1.0965 e+02$ & $5.8827 e+02$ & ; & 04 & $-\mathrm{s} 1$ & - N1 \\
\hline 8 & 9 & 10 & 1 & $1.1910 \mathrm{e}+02$ & $5.9287 e+02$ & ; & N1 & $-\mathrm{s} 2$ & -01 \\
\hline 8 & 9 & 11 & 1 & $1.1910 \mathrm{e}+02$ & $5.9287 e+02$ & ; & N1 & $-\mathrm{s} 2$ & -02 \\
\hline 8 & 9 & 12 & 1 & $1.0819 e+02$ & $1.3623 e+02$ & ; & N1 & $-\mathrm{s} 2$ & $-\mathrm{C} 2$ \\
\hline 9 & 12 & 13 & 1 & $1.0968 \mathrm{e}+02$ & $5.2819 e+02$ & ; & $\mathrm{s} 2$ & $-\mathrm{C} 2$ & - F5 \\
\hline 9 & 12 & 14 & 1 & $1.0968 e+02$ & $5.2819 e+02$ & ; & $\mathrm{s} 2$ & $-\mathrm{C} 2$ & $-F 6$ \\
\hline 9 & 12 & 15 & 1 & $1.0968 \mathrm{e}+02$ & $5.2819 e+02$ & ; & $\mathrm{s} 2$ & $-\mathrm{C} 2$ & - F4 \\
\hline 10 & 9 & 11 & 1 & $1.2005 e+02$ & $6.1580 e+02$ & ; & 01 & $-\mathrm{s} 2$ & -02 \\
\hline 10 & 9 & 12 & 1 & $1.0861 \mathrm{e}+02$ & $5.4702 \mathrm{e}+02$ & ; & 01 & $-\mathrm{s} 2$ & $-\mathrm{C} 2$ \\
\hline 11 & 9 & 12 & 1 & $1.0861 \mathrm{e}+02$ & $5.4702 e+02$ & ; & $\mathrm{O} 2$ & $-\mathrm{s} 2$ & $-\mathrm{C} 2$ \\
\hline 13 & 12 & 14 & 1 & $1.0736 \mathrm{e}+02$ & $5.9321 e+02$ & ; & F5 & $-\mathrm{C} 2$ & $-F 6$ \\
\hline 13 & 12 & 15 & 1 & $1.0736 \mathrm{e}+02$ & $5.9321 \mathrm{e}+02$ & ; & F5 & $-\mathrm{C} 2$ & - F4 \\
\hline 14 & 12 & 15 & 1 & $1.0736 e+02$ & $5.9321 e+02$ & & $\mathrm{~F} 6$ & $-\mathrm{C}_{2}$ & - F4 \\
\hline
\end{tabular}




\begin{tabular}{|c|c|c|c|c|c|c|c|c|c|c|c|c|c|c|c|}
\hline$i$ & j & $\begin{array}{c}\text { proper } \\
k\end{array}$ & 1 & func & $\mathrm{CO}$ & $\mathrm{C} 1$ & $\mathrm{C} 2$ & C3 & $\mathrm{C} 4$ & C5 & & & & & \\
\hline 1 & 2 & 5 & 6 & 3 & 0.60436 & 1.81307 & 0.00000 & -2.41742 & 0.00000 & 0.00000 & ; & F1- & $\mathrm{C} 1-$ & S1- & 03 \\
\hline 1 & 2 & 5 & 7 & 3 & 0.60436 & 1.81307 & 0.00000 & -2.41742 & 0.00000 & 0.00000 & ; & F1- & C1- & S1- & 04 \\
\hline 1 & 2 & 5 & 8 & 3 & 0.60436 & 1.81307 & 0.00000 & -2.41742 & 0.00000 & 0.00000 & ; & F1- & C1- & S1- & N1 \\
\hline 2 & 5 & 8 & 9 & 3 & 30.54320 & 22.17520 & 0.00000 & 8.36800 & 0.00000 & 0.00000 & ; & C1- & S1- & N1- & S2 \\
\hline 3 & 2 & 5 & 6 & 3 & 0.60436 & 1.81307 & 0.00000 & -2.41742 & 0.00000 & 0.00000 & ; & F2- & C1- & S1- & 03 \\
\hline 3 & 2 & 5 & 7 & 3 & 0.60436 & 1.81307 & 0.00000 & -2.41742 & 0.00000 & 0.00000 & ; & F2- & C1- & S1- & 04 \\
\hline 3 & 2 & 5 & 8 & 3 & 0.60436 & 1.81307 & 0.00000 & -2.41742 & 0.00000 & 0.00000 & ; & F2- & C1- & S1- & N1 \\
\hline 4 & 2 & 5 & 6 & 3 & 0.60436 & 1.81307 & 0.00000 & -2.41742 & 0.00000 & 0.00000 & ; & F3- & C1- & S1- & 03 \\
\hline 4 & 2 & 5 & 7 & 3 & 0.60436 & 1.81307 & 0.00000 & -2.41742 & 0.00000 & 0.00000 & ; & F3- & C1- & S1- & 04 \\
\hline 4 & 2 & 5 & 8 & 3 & 0.60436 & 1.81307 & 0.00000 & -2.41742 & 0.00000 & 0.00000 & ; & F3- & C1- & S1- & N1 \\
\hline 5 & 8 & 9 & 10 & 3 & 55.78946 & 0.00000 & -55.78946 & 0.00000 & 0.00000 & 0.00000 & ; & S1- & N1- & S2- & 01 \\
\hline 5 & 8 & 9 & 11 & 3 & 55.78946 & 0.00000 & -55.78946 & 0.00000 & 0.00000 & 0.00000 & ; & S1- & N1- & S2- & $\mathrm{O} 2$ \\
\hline 5 & 8 & 9 & 12 & 3 & 55.78946 & 0.00000 & -55.78946 & 0.00000 & 0.00000 & 0.00000 & ; & S1- & N1- & S2- & $\mathrm{C} 2$ \\
\hline 6 & 5 & 8 & 9 & 3 & 30.54320 & 22.17520 & 0.00000 & 8.36800 & 0.00000 & 0.00000 & ; & O3- & S1- & N1- & $\mathrm{S} 2$ \\
\hline 7 & 5 & 8 & 9 & 3 & 30.54320 & 22.17520 & 0.00000 & 8.36800 & 0.00000 & 0.00000 & ; & O4- & S1- & N1- & S2 \\
\hline 8 & 9 & 12 & 13 & 3 & 0.60436 & 1.81307 & 0.00000 & -2.41742 & 0.00000 & 0.00000 & ; & N1- & S2- & C2- & F5 \\
\hline 8 & 9 & 12 & 14 & 3 & 0.60436 & 1.81307 & 0.00000 & -2.41742 & 0.00000 & 0.00000 & ; & N1- & S2- & C2- & F6 \\
\hline 8 & 9 & 12 & 15 & 3 & 0.60436 & 1.81307 & 0.00000 & -2.41742 & 0.00000 & 0.00000 & ; & N1- & S2- & C2- & F4 \\
\hline 10 & 9 & 12 & 13 & 3 & 0.60436 & 1.81307 & 0.00000 & -2.41742 & 0.00000 & 0.00000 & ; & O1- & S2- & C2- & F5 \\
\hline 10 & 9 & 12 & 14 & 3 & 0.60436 & 1.81307 & 0.00000 & -2.41742 & 0.00000 & 0.00000 & ; & O1- & S2- & C2- & F6 \\
\hline 10 & 9 & 12 & 15 & 3 & 0.60436 & 1.81307 & 0.00000 & -2.41742 & 0.00000 & 0.00000 & ; & O1- & S2- & C2- & F4 \\
\hline 11 & 9 & 12 & 13 & 3 & 0.60436 & 1.81307 & 0.00000 & -2.41742 & 0.00000 & 0.00000 & ; & $02-$ & S2- & C2- & F5 \\
\hline 11 & 9 & 12 & 14 & 3 & 0.60436 & 1.81307 & 0.00000 & -2.41742 & 0.00000 & 0.00000 & ; & O2- & S2- & C2- & F6 \\
\hline 11 & 9 & 12 & 15 & 3 & 0.60436 & 1.81307 & 0.00000 & -2.41742 & 0.00000 & 0.00000 & ; & O2- & S2- & C2- & F4 \\
\hline
\end{tabular}

ADN

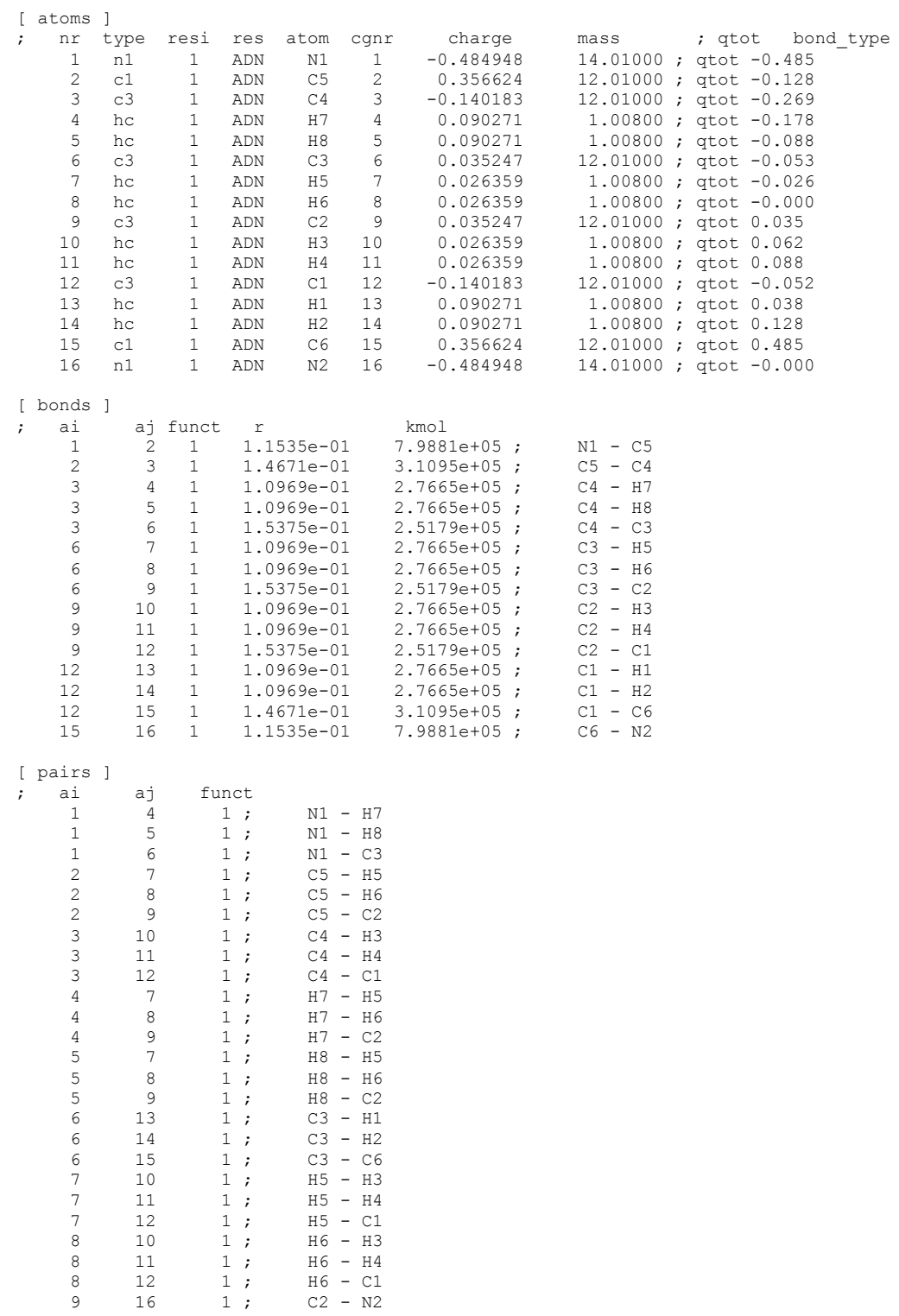




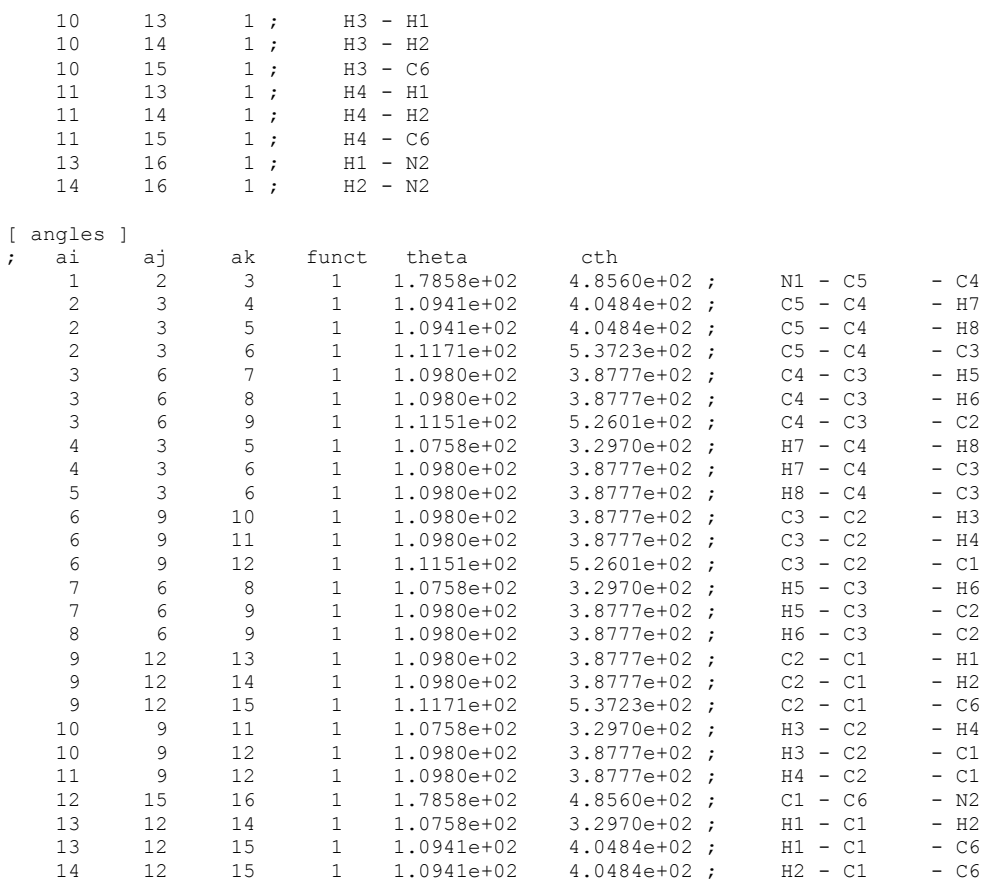

[ dihedrals ] ; propers

; $\begin{array}{rrr}\text { i } & j & \mathrm{k} \\ 1 & 2 & 3 \\ 1 & 2 & 3 \\ 1 & 2 & 3 \\ 2 & 3 & 6 \\ 2 & 3 & 6 \\ 2 & 3 & 6 \\ 3 & 6 & 9 \\ 3 & 6 & 9 \\ 3 & 6 & 9 \\ 4 & 3 & 6 \\ 4 & 3 & 6 \\ 4 & 3 & 6 \\ 5 & 3 & 6 \\ 5 & 3 & 6 \\ 5 & 3 & 6 \\ 6 & 9 & 12 \\ 6 & 9 & 12 \\ 6 & 9 & 12 \\ 7 & 6 & 9 \\ 7 & 6 & 9 \\ 7 & 6 & 9 \\ 8 & 6 & 9 \\ 8 & 6 & 9 \\ 8 & 6 & 9 \\ 9 & 12 & 15 \\ 10 & 9 & 12 \\ 10 & 9 & 12 \\ 10 & 9 & 12 \\ 11 & 9 & 12 \\ 11 & 9 & 12 \\ 11 & 9 & 12 \\ 13 & 12 & 15 \\ 14 & 12 & 15 \\ & & \end{array}$

$\begin{array}{rcccc}\text { to use combine multiple AMBER } & \text { torsions } & \text { per quartet } \\ \text { func } & \text { C0 } & \text { C1 } & \text { C2 } & \text { C3 } \\ 3 & 0.00000 & 0.00000 & 0.00000 & 0.00000 \\ 3 & 0.00000 & 0.00000 & 0.00000 & 0.00000 \\ 3 & 0.00000 & 0.00000 & 0.00000 & 0.00000 \\ 3 & 0.65084 & 1.95253 & 0.00000 & -2.60338 \\ 3 & 0.65084 & 1.95253 & 0.00000 & -2.60338 \\ 3 & 0.65084 & 1.95253 & 0.00000 & -2.60338 \\ 3 & 0.66944 & 2.00832 & 0.00000 & -2.67776 \\ 3 & 0.66944 & 2.00832 & 0.00000 & -2.67776 \\ 3 & 3.68192 & 3.09616 & -2.09200 & -3.01248 \\ 3 & 0.62760 & 1.88280 & 0.00000 & -2.51040 \\ 3 & 0.62760 & 1.88280 & 0.00000 & -2.51040 \\ 3 & 0.66944 & 2.00832 & 0.00000 & -2.67776 \\ 3 & 0.62760 & 1.88280 & 0.00000 & -2.51040 \\ 3 & 0.62760 & 1.88280 & 0.00000 & -2.51040 \\ 3 & 0.66944 & 2.00832 & 0.00000 & -2.67776 \\ 3 & 0.66944 & 2.00832 & 0.00000 & -2.67776 \\ 3 & 0.66944 & 2.00832 & 0.00000 & -2.67776 \\ 3 & 0.65084 & 1.95253 & 0.00000 & -2.60338 \\ 3 & 0.62760 & 1.88280 & 0.00000 & -2.51040 \\ 3 & 0.62760 & 1.88280 & 0.00000 & -2.51040 \\ 3 & 0.66944 & 2.00832 & 0.00000 & -2.67776 \\ 3 & 0.62760 & 1.88280 & 0.00000 & -2.51040 \\ 3 & 0.62760 & 1.88280 & 0.00000 & -2.51040 \\ 3 & 0.66944 & 2.00832 & 0.00000 & -2.67776 \\ 3 & 0.00000 & 0.00000 & 0.00000 & 0.00000 \\ 3 & 0.62760 & 1.88280 & 0.00000 & -2.51040 \\ 3 & 0.62760 & 1.88280 & 0.00000 & -2.51040 \\ 3 & 0.65084 & 1.95253 & 0.00000 & -2.60338 \\ 3 & 0.62760 & 1.88280 & 0.00000 & -2.51040 \\ 3 & 0.62760 & 1.88280 & 0.00000 & -2.51040 \\ 3 & 0.65084 & 1.95253 & 0.00000 & -2.60338 \\ 3 & 0.00000 & 0.00000 & 0.00000 & 0.00000 \\ 3 & 0.00000 & 0.00000 & 0.00000 & 0.00000\end{array}$

$\mathrm{C} 4$

0.00000 0.00000 0.00000 0.00000 0.00000 0.00000 0.00000 0.00000 0.0000 0.0000 0.00000 0.00000 0.00000 0.00000 0.00000 0.00000 0.00000 0.00000 0.00000 0.00000 0.0000 0.00000 0.00000 0.00000 0.00000 0.00000 0.00000 0.00000 0.00000 0.00000 0.00000 0.00000 0.00000 0.00000 0.00000
C5

$0.00000 ; \quad \mathrm{N} 1-\quad \mathrm{C} 5-\quad \mathrm{C} 4-\quad \mathrm{H} 7$ $0.00000 ; \quad \mathrm{N} 1-\quad \mathrm{C} 5-\quad \mathrm{C} 4-\quad \mathrm{H} 8$ $\begin{array}{lllll}0.00000 ; & \mathrm{N} 1- & \mathrm{C} 5- & \mathrm{C} 4- & \mathrm{C} 3 \\ 0.00000 ; & \mathrm{C} 5- & \mathrm{C} 4- & \mathrm{C} 3- & \mathrm{H} 5\end{array}$ $0.00000 ; \quad \mathrm{C} 5-\quad \mathrm{C} 4-\quad \mathrm{C} 3-\quad \mathrm{H} 6$ $0.00000 ; \quad \mathrm{C} 5-\quad \mathrm{C} 4-\quad \mathrm{C} 3-\quad \mathrm{C} 2$

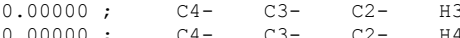
$0.00000 ; \quad \mathrm{C} 4-\mathrm{C} 3-\mathrm{C}_{2}-\mathrm{C}^{-}$ $0.00000 ; \quad \mathrm{C} 7-\mathrm{C} 4-\mathrm{C} 3-2 \mathrm{C} 5$ $0.00000 ; \quad \mathrm{H} 7-\mathrm{C}_{-}-\mathrm{C}^{-} \mathrm{H} 5$ $0.00000 ; \quad \mathrm{H} 7-\mathrm{C} 4-\mathrm{C} 3-\mathrm{C} 2$ $0.00000 ; \quad \mathrm{H} 8-\mathrm{C} 4-\mathrm{C} 3-\mathrm{H} 5$ $0.00000 ; \mathrm{H} 8-\mathrm{C} 4-\mathrm{C} 3-\mathrm{H} 6$ $\begin{array}{lllll}0.00000 ; & \mathrm{H} 8- & \mathrm{C} 4- & \mathrm{C} 3- & \mathrm{C} 2 \\ 0.00000 ; & \mathrm{C} 3- & \mathrm{C} 2- & \mathrm{C} 1- & \mathrm{H} 1\end{array}$ $0.00000 ; \quad \mathrm{C} 3-\quad \mathrm{C} 2-\mathrm{C} 1-\quad \mathrm{H} 2$ $0.00000 ; \quad \mathrm{C} 3-\quad \mathrm{C} 2-\quad \mathrm{C} 1-\quad \mathrm{C} 6$ $\begin{array}{lllll}0.00000 ; & \mathrm{H} 5- & \mathrm{C} 3- & \mathrm{C} 2- & \mathrm{H} 3 \\ 0.00000 ; & \mathrm{H} 5- & \mathrm{C} 3- & \mathrm{C} 2- & \mathrm{H} 4\end{array}$

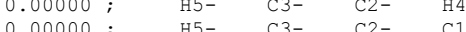
$\begin{array}{lllll}0.00000 ; & \mathrm{H} 5- & \mathrm{C} 3- & \mathrm{C} 2- & \mathrm{C} 1 \\ 0.00000 ; & \mathrm{H} 6- & \mathrm{C} 3- & \mathrm{C} 2- & \mathrm{H} 3\end{array}$ $0.00000 ; \quad \mathrm{H} 6-\quad \mathrm{C} 3-\quad \mathrm{C} 2-\quad \mathrm{H} 4$ $0.00000 ; \quad \mathrm{H} 6-\quad \mathrm{C} 3-\quad \mathrm{C} 2-\mathrm{C} 1$ $0.00000 ; \quad \mathrm{C} 2-\mathrm{C} 1-\quad \mathrm{C} 6-\quad \mathrm{N} 2$ $\begin{array}{lllll}0.00000 ; & \mathrm{H} 3- & \mathrm{C} 2- & \mathrm{C} 1- & \mathrm{H} 1 \\ 0.00000 ; & \mathrm{H} 3- & \mathrm{C} 2- & \mathrm{C} 1- & \mathrm{H} 2\end{array}$ $0.00000 ; \quad \mathrm{H} 3-\quad \mathrm{C} 2-\quad \mathrm{C} 1-\quad \mathrm{C} 6$ $\begin{array}{lllll}0.00000 ; & \mathrm{H} 4- & \mathrm{C} 2- & \mathrm{C} 1- & \mathrm{H} 1 \\ 0.00000 & \mathrm{H} 4- & \mathrm{C} 2- & \mathrm{C} 1- & \mathrm{H} 2\end{array}$ $\begin{array}{lllll}0.00000 ; & \mathrm{H} 4- & \mathrm{C} 2- & \mathrm{C} 1- & \mathrm{H} 2 \\ 0.00000 ; & \mathrm{H} 4- & \mathrm{C} 2- & \mathrm{C} 1- & \mathrm{C} 6\end{array}$ $\begin{array}{lllll}0.00000 ; & \mathrm{H} 4- & \mathrm{C} 2- & \mathrm{C} 1- & \mathrm{C} 6 \\ 0.00000 ; & \mathrm{H} 1- & \mathrm{C} 1- & \mathrm{C} 6- & \mathrm{N} 2\end{array}$

\section{GLN}

\begin{tabular}{|c|c|c|c|c|c|c|c|c|c|}
\hline \multicolumn{10}{|c|}{ atoms ] } \\
\hline $\mathrm{nr}$ & type & resi & res & atom & $\operatorname{cgn} r$ & charge & mass & ; qtot & bond type \\
\hline 1 & $\mathrm{n} 1$ & 1 & GLN & N1 & 1 & -0.471701 & 14.01000 & ; qtot & $-0.472--1$ \\
\hline 2 & c1 & 1 & GLN & $\mathrm{C} 4$ & 2 & 0.342369 & 12.01000 & ; qtot & -0.129 \\
\hline 3 & c3 & 1 & GLN & C3 & 3 & -0.140507 & 12.01000 & ; qtot & -0.270 \\
\hline 4 & he & 1 & GLN & H5 & 4 & 0.094445 & 1.00800 & ; qtot & -0.175 \\
\hline 5 & he & 1 & GLN & H6 & 5 & 0.094445 & 1.00800 & ; qtot & -0.081 \\
\hline 6 & c3 & 1 & GLN & C2 & 6 & 0.081522 & 12.01000 & ; qtot & 0.001 \\
\hline 7 & hc & 1 & GLN & H3 & 7 & 0.040188 & 1.00800 & ; qtot & 0.041 \\
\hline 8 & he & 1 & GLN & H4 & 8 & 0.040188 & 1.00800 & ; qtot & 0.081 \\
\hline 9 & c3 & 1 & GLN & $\mathrm{C} 1$ & 9 & -0.140507 & 12.01000 & ; qtot & -0.060 \\
\hline 10 & he & 1 & GLN & H1 & 10 & 0.094445 & 1.00800 & ; qtot & 0.035 \\
\hline 11 & he & 1 & GLN & H2 & 11 & 0.094445 & 1.00800 & ; qtot & 0.129 \\
\hline 12 & c1 & 1 & GLN & C5 & 12 & 0.342369 & 12.01000 & ; qtot & 0.472 \\
\hline 13 & n1 & 1 & GLN & N2 & 13 & -0.471701 & 14.01000 & gtot. & -0.000 \\
\hline
\end{tabular}

[ bonds ] 


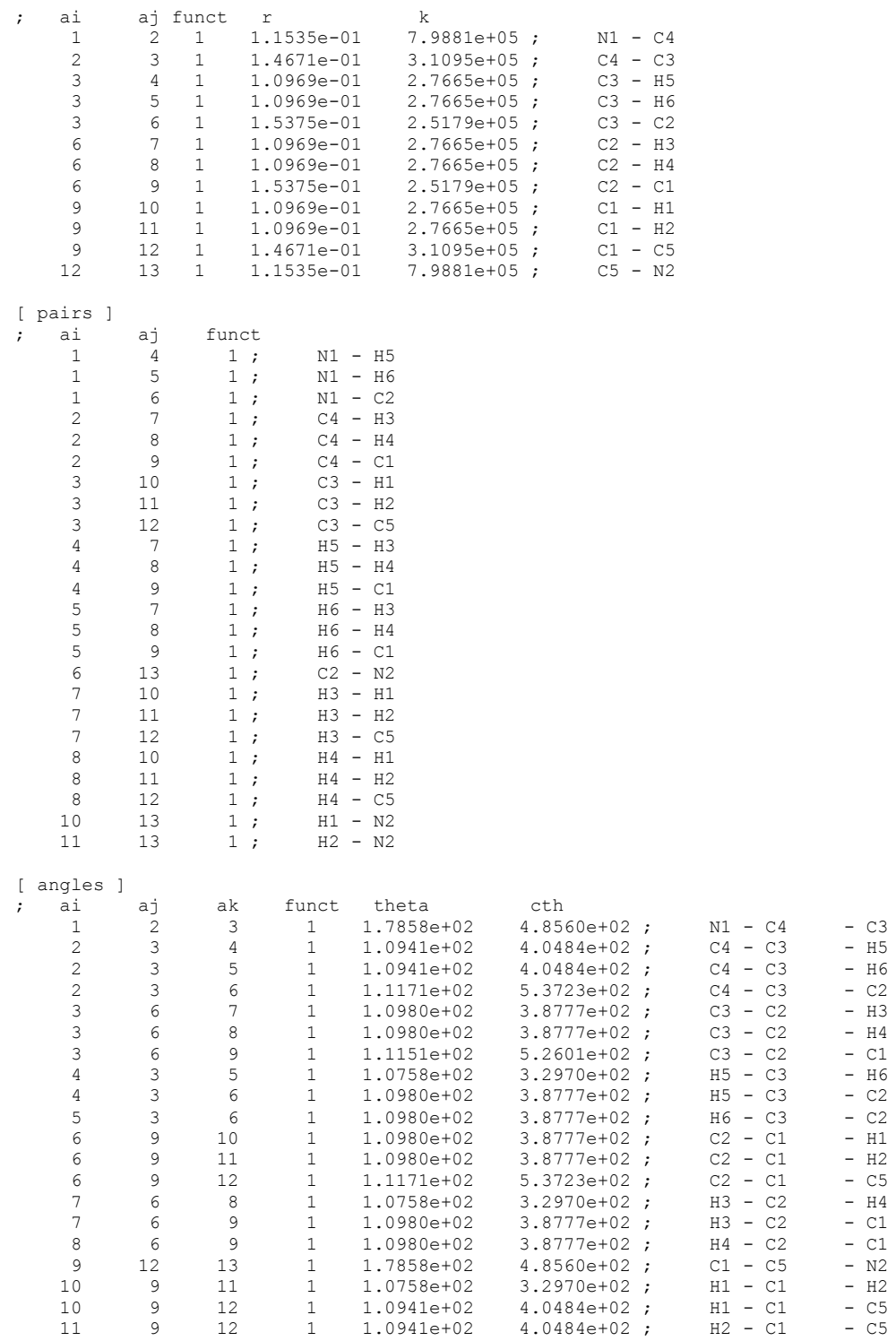

[ dihedrals ] ; propers

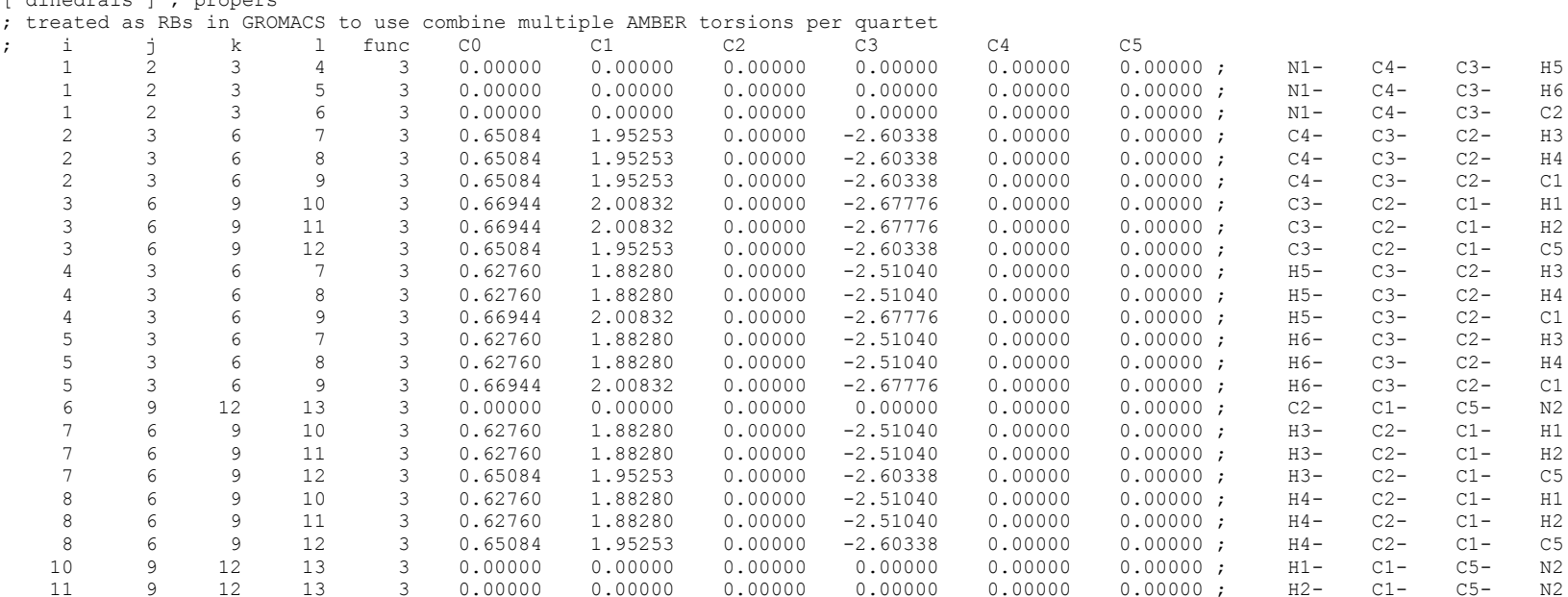

